\title{
On the coupling between molecular diffusion and solvation shell exchange
}

\author{
Møller, Klaus Braagaard; Rey, Rossend; Masia, Marco; Hynes, James T.
}

Published in:

Journal of Chemical Physics

Link to article, DOI:

$10.1063 / 1.1863172$

Publication date:

2005

Document Version

Publisher's PDF, also known as Version of record

Link back to DTU Orbit

Citation (APA):

Møller, K. B., Rey, R., Masia, M., \& Hynes, J. T. (2005). On the coupling between molecular diffusion and solvation shell exchange. Journal of Chemical Physics, 122(11), 114508. https://doi.org/10.1063/1.1863172

\section{General rights}

Copyright and moral rights for the publications made accessible in the public portal are retained by the authors and/or other copyright owners and it is a condition of accessing publications that users recognise and abide by the legal requirements associated with these rights.

- Users may download and print one copy of any publication from the public portal for the purpose of private study or research.

- You may not further distribute the material or use it for any profit-making activity or commercial gain

- You may freely distribute the URL identifying the publication in the public portal 


\title{
On the coupling between molecular diffusion and solvation shell exchange
}

\author{
Klaus B. Møller \\ Department of Chemistry, Technical University of Denmark, Building 207, 2800 Kgs. Lyngby, Denmark \\ Rossend Rey and Marco Masia \\ Departament de Física i Enginyeria Nuclear, Universitat Politècnica de Catalunya, Campus Nord B4-B5, \\ Barcelona 08034, Spain \\ James T. Hynes \\ Département de Chimie, Ecole Normale Supérieure, 24 rue Lhomond, 75231 Paris Cedex 5, France and \\ Department of Chemistry and Biochemistry University of Colorado at Boulder, \\ Boulder, Colorado 80309
}

(Received 13 September 2004; accepted 6 January 2005; published online 21 March 2005)

\begin{abstract}
The connection between diffusion and solvent exchanges between first and second solvation shells is studied by means of molecular dynamics simulations and analytic calculations, with detailed illustrations for water exchange for the $\mathrm{Li}^{+}$and $\mathrm{Na}^{+}$ions, and for liquid argon. First, two methods are proposed which allow, by means of simulation, to extract the quantitative speed-up in diffusion induced by the exchange events. Second, it is shown by simple kinematic considerations that the instantaneous velocity of the solute conditions to a considerable extent the character of the exchanges. Analytic formulas are derived which quantitatively estimate this effect, and which are of general applicability to molecular diffusion in any thermal fluid. Despite the simplicity of the kinematic considerations, they are shown to well describe many aspects of solvent exchange/ diffusion coupling features for nontrivial systems. () 2005 American Institute of Physics.
\end{abstract}

[DOI: $10.1063 / 1.1863172]$

\section{INTRODUCTION}

Although we are close to the centennial of the first theoretical studies on molecular diffusion ${ }^{1}$ this fundamental process is rich enough to constitute an area of active research for years to come. While the subject has extended in many interesting directions, here we revisit some not fully understood features of diffusion in thermal fluids. In these conditions the basic picture is one in which the diffusing molecule (hereinafter referred to as the solute) undergoes random collisions which hinder its motion, and result in a mean square displacement only linear in time. It is interesting to note that the role and fate of the colliding neighbors (solvation molecules) tend to be often overlooked, as in most instances the interaction is feeble and only coarse-grained aspects (such as solute size and solvent viscosity) are considered to be of relevance. The remarkable numerical success of the macroscopic Stokes law down to the atomic scale for a large variety of systems ${ }^{2}$ (including a prominent example such as liquid water self diffusion ${ }^{3}$ ) might be taken as a reflection of the unimportance of the neighbors dynamics, even when the sizes of the solute and the solvent molecules are comparable. However, it has been argued that microscopic aspects of diffusion are in fact important. ${ }^{4,5} \mathrm{~A}$ particular instance in which this Stokes-Einstein macroscopic approach is generally agreed to fail qualitatively is that of ionic diffusion (of evident interest if taking place in water), where for instance an increase of the diffusion coefficient with decreasing radius of the ion is not observed, ${ }^{6}$ while it is a direct prediction of the macroscopic theory. The qualitative explanation ${ }^{7,8}$ focuses on the nature of the electrostatic interaction, which for the ion in a polar solvent case is strong enough (specially for the smaller ions) so that the solvation molecules follow the ion motion in time, with occasional exchanges between solvation shells: one molecule from the first solvation shell escapes to the second shell while a second shell molecule enters into the immediate vicinity of the ion (both events being simultaneous or asynchronous, with no general priority rule in the latter case). The increased drag on the ion exerted by this cohort of nearest neighbors explains the breakdown of the macroscopic approach, with a diffusion constant lower than what should be expected for the bare ionic radius. Nevertheless, in the strong interaction limit (small ionic radius and/or high charge, so that the first shell solvation molecules do not undergo any exchange) the macroscopic theory regains at least its numerical validity, as applied to the complex defined by the ion plus nearest neighbors (an approach known as the "solventberg" model).

The previous considerations highlight the potential role of the exchanges for the diffusion process in what might be called the intermediate regime, where the dynamics of the solvation shell molecules is highly correlated with that of the solute and yet, the exchanges are still not rare (on the time scale for diffusion). We believe that in this scenario the effect of the solvation shell exchange is not fully understood. Actually, not even the exchange process by itself can be regarded as a solved issue: although the exchange times for ions in solution have been the subject of study for quite some time $e^{6,9-12}$ (by means of molecular dynamics simulations), the mechanisms and stereochemistry of the exchange process are just starting to be scrutinized, ${ }^{13-16}$ usually motivated by its key role in other important processes such as ion reactivity. To evidence some unclear aspects of the influence of ex- 
change on diffusion with an example, an issue such as a characterization of the quantitative speed-up in diffusion induced by exchanges remains unaddressed. Moreover, it is known that the exchange times for ions in water are typically larger than (roughly) 10 ps (Ref. 10) while the time span required to obtain the diffusion coefficient with a high degree of accuracy (from the mean square displacement or from the velocity correlation function) is of the order of $1 \mathrm{ps}$. It is then somewhat puzzling that the solventberg picture is not mandatory in all cases, given that on the time scale required to reach a diffusive behavior there seem to be no exchanges in either case (as for a multiple charged ion). These questions constitute a first topic of attention in this work.

The main line of study, though, will focus on the inverse problem, namely, on the possible influence of diffusion on solvation shell exchange. The basic question here is if a given exchange event is conditioned in some way by the instantaneous state of motion of the solute, what we believe constitutes a rather new approach on this issue. At first sight, it might be thought that any such effect should be feeble. To start with, it is easy to show that exchanges are independent of diffusion in some instances: in the simple case of a massive particle (compared with the solvent molecules' mass), there will be many exchanges occurring while there is almost no diffusion of the solvated particle. In addition, these exchanges will obviously take place with a (very nearly) symmetric distribution around the solute so that no correlation will exist with its motion. Certainly this is a limiting case, but if we consider for instance the (random) oscillatory motion of an ion inside its cage of (first shell) solvating molecules while the whole complex diffuses, and that exchanges are rather infrequent, a correlation of the instantaneous ion velocity with the exchange between a second shell molecule and a first shell one may not seem likely a priori. Remarkably, it will be shown within that an important aspect of this problem allows for an exact analytical approach, which should apply to any thermal fluid. It so happens that the stereochemistry of the exchanges is effectively driven (in a probabilistic way) by the instantaneous velocity of the solute: the exchange events occur according to a nonsymmetric probability distribution around this direction, the counterexample starting this paragraph being a limiting (symmetric) case. While most of the results presented to illustrate these issues will correspond to the particular case of ionic diffusion in water (selected both for its relevance as well as for computational convenience), given the general character of the previous considerations, examples corresponding to simple liquids will also be included for the sake of completeness.

The outline of the paper is as follows. A summary of the simulation details is given in the following section. The results and discussion on the influence of solvation shell exchange on diffusion are presented in Sec. III, while the inverse problem (the influence of diffusion on the exchange process) is addressed in Sec. IV. The main conclusions are summarized in Sec. V. An Appendix is also included, with the details of the derivation of some analytical expressions used in the main text.

\section{COMPUTATIONAL DETAILS}

As just described most of the molecular dynamics (MD) simulations correspond to single ions dissolved in water. The simulated systems consist of an ion $\left(\mathrm{Li}^{+}\right.$or $\left.\mathrm{Na}^{+}\right)$plus 215 water molecules in a cubic box with standard periodic conditions. The water model is SPC (Ref. 17) (if not stated otherwise), keeping the water molecules rigid via the SHAKE algorithm. ${ }^{18}$ The ion-water interaction parameters for ions are those of Ref. 19 for sodium and those of Ref. 20 for lithium. Long-range forces were computed by the Ewald summation method, ${ }^{21}$ and a leap-frog integration algorithm with coupling to a thermal bath ${ }^{22}$ has been used, with a $1 \mathrm{fs}$ time step, and the value of the coupling set to $0.1 \mathrm{ps}$.

A second set of simulations has also been done for pure simple liquids (Argon at liquid conditions), with the interaction parameters taken from Refs. 23 and 24. A leap-frog integration algorithm has been used without thermal control and with a time step of $5 \mathrm{fs}$. Periodic boundary conditions for $500 \mathrm{Ar}$ atoms in a cubic box were applied.

\section{EFFECT OF SOLVATION SHELL EXCHANGE ON DIFFUSION}

As previously described in the Introduction, within the regime of interest the first solvation shell follows the solute in its diffusive motion, with the exchange events being relatively uncommon. Thus, we seek to connect two phenomena that take place on rather different time scales, and which consequently are usually studied with different tools. Diffusion is usually addressed with long (typically hundreds of picosecond) equilibrium runs, from which the mean square displacement (MSD) is computed up to a certain time limit (much shorter than the total simulation time): for ionic diffusion in liquid water the typical length required for the MSD is of substantially less than 10 ps, but certainly longer than $\approx 1 \mathrm{ps}$, in order to get a sufficiently accurate value of the diffusion coefficient $D$. On the other hand, the onset and completion of an exchange event last typically less than $1 \mathrm{ps}$, so that exchanges must be studied with short runs starting from properly selected initial conditions. ${ }^{13,15}$ These time scales suggest that asking for instance about the value of the diffusion coefficient during an exchange does not seem to be meaningful, since the exchange event lasts less than the time required to observe diffusive behavior in the MSD. Nevertheless, an indirect method is possible to study the signature of exchanges on diffusion: the portions of the long equilibrium run during which no exchanges take place are used to compute a new MSD function (and a new velocity selfcorrelation function), from which a different diffusion coefficient results $\left(D^{\prime}\right)$. It should be obvious that the effect of the exchanges on diffusion will be reflected in a quantitative difference between $D$ and $D^{\prime}$ : one should expect that $D$ is in all cases larger than $D^{\prime}$, as the latter corresponds to the first shell moving concertedly with the ion, with no exchanges. Since it is only needed that the aforementioned portions have a length of some $10 \mathrm{ps}$, and exchanges occur on this time scale or longer, ${ }^{10}$ the computation of $D^{\prime}$ is perfectly feasible from a statistical point of view. The interesting feature of this simple approach is that, to our knowledge, it will provide the 

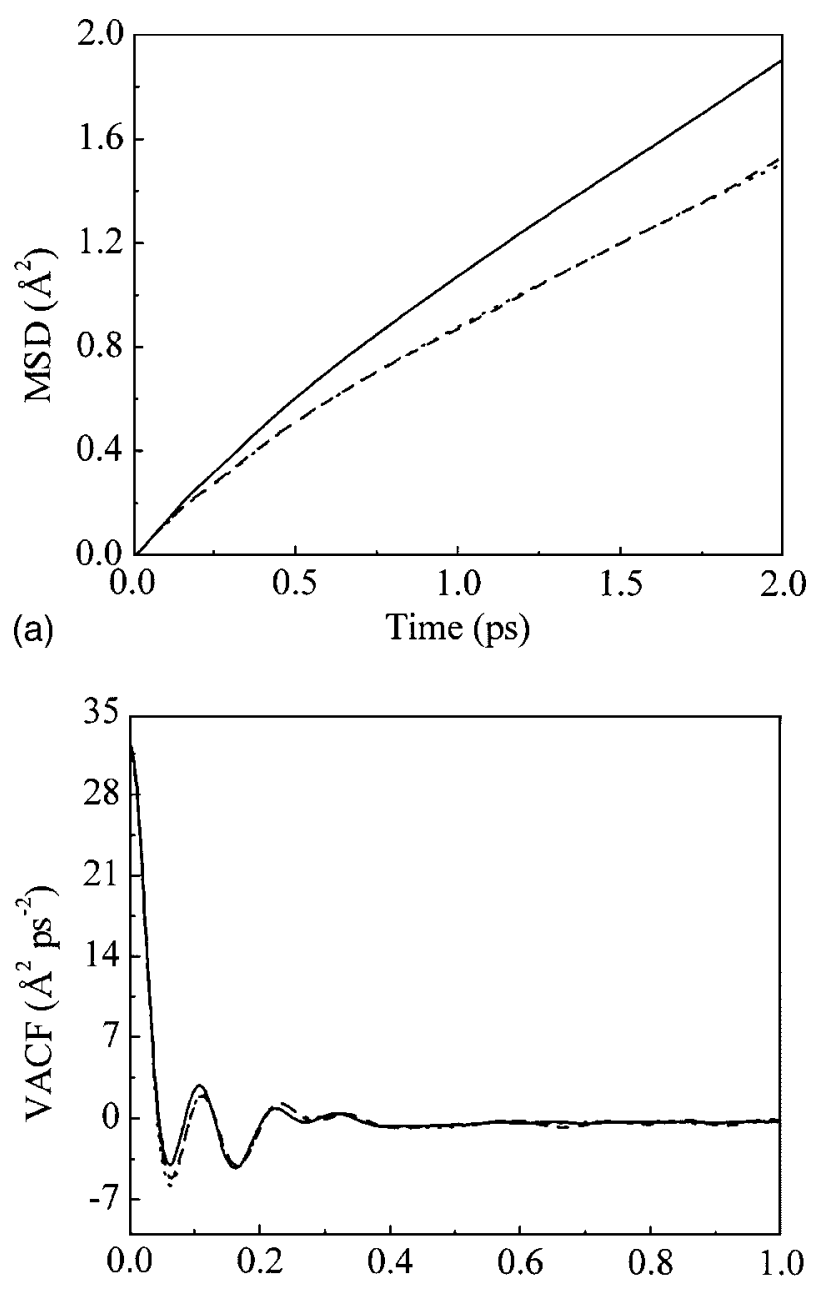

(b)

Time (ps)

FIG. 1. $\mathrm{Na}^{+}$diffusion in ambient liquid water. Solid line, results from equilibrium simulation; dashed line, results only including portions of equilibrium run without exchanges; points, results from simulations including an effective "wall" between first and second hydration shells. (a) Ion mean square displacement; (b) ion velocity self-correlation function.

first quantitative estimation on the effect of exchanges: although, as argued in the Introduction, it is generally accepted that diffusion is slower if no exchanges take place, the extent of this slowdown is unknown.

We have first addressed the case of $\mathrm{Na}^{+}$in liquid water, a system for which kinetic ${ }^{13}$ and dynamic ${ }^{14}$ characteristics of the exchanges have been studied in detail. The MSD and velocity self-correlation functions have been computed in the manner just described, from a run of 3 ns. Figure 1(a) displays the results for the MSD obtained from the whole simulation, together with the one obtained from trajectories with no exchanges. As expected, the latter has a clearly smaller slope, consistent with a slower diffusion, with the actual values of the diffusion coefficients being $D=1.37$ in front of $D^{\prime}=1.05$ (both in units of $10^{-5} \mathrm{~cm}^{2} / \mathrm{s}$ ). We see that exchanges increase the diffusion coefficient by $30 \%$ with respect to the value obtained if exchanges do not occur, which constitutes a basic result of this work. Identical results for the diffusion coefficients are obtained from the velocity selfcorrelation functions obtained in each case. The qualitative differences between the latter functions are evident in Fig.

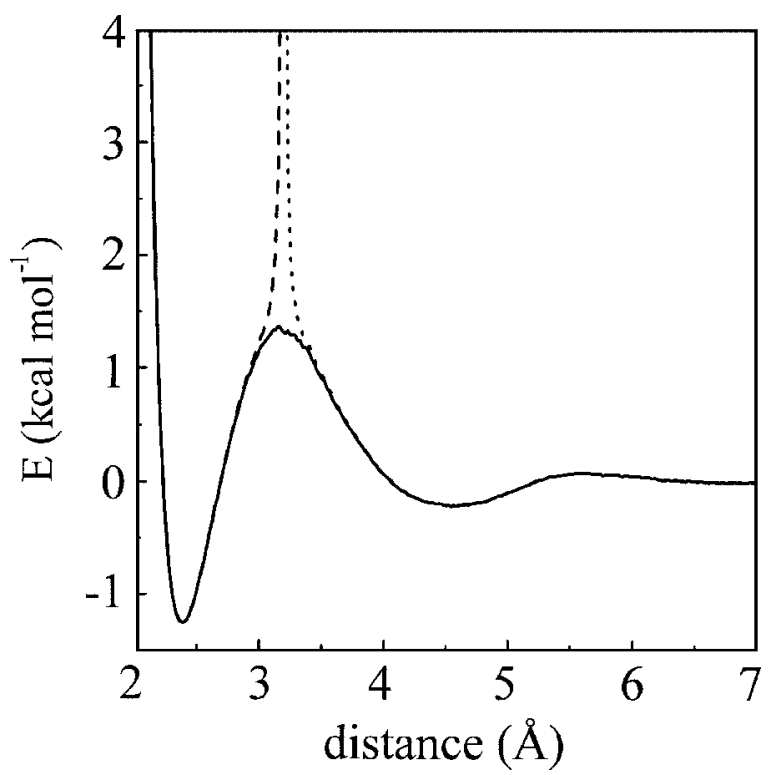

FIG. 2. Solid line, potential of mean force [Eq. (3)] between $\mathrm{Na}^{+}$and a water molecule; dashed line, same plus the contribution of $V_{\text {in }(r)}$ [Eq. (1)]; points, same plus the contribution of $V_{\text {out }}(r)[\mathrm{Eq}$. (2)].

1(b): the initial backscattering to negative values is stronger if no exchanges occur, which can be understood considering that in this case the ion is at all times constrained to an oscillatory motion within the cage of first neighbors.

A second independent methodology has also been devised to double check the previous results. The interaction between the ion and its solvation molecules is altered in order to preclude any exchange between first and second shells, while trying to minimally alter other properties of the system. The ion-oxygen interaction potential for water molecules initially within the first shell is augmented by a term

$$
V_{\text {in }}(r)=\beta e^{\alpha\left(r-r^{\ddagger}\right)},
$$

while for those molecules initially outside the first shell we use

$$
V_{\text {out }}(r)=\beta e^{-\alpha\left(r-r^{\dot{亠}}\right)} .
$$

Both contributions together establish a sort of "wall" between first and second shells, which effectively results in the absence of exchanges. The parameters are chosen so that this wall has a fairly short range, $\alpha=10 \AA^{-1}$ and $\beta=5 \mathrm{~kJ} / \mathrm{mol}$, with $r^{\ddagger}$ corresponding to the limit of the first hydration shell $\left(r^{\ddagger} \approx 3.2 \AA\right)$. The effect of these new potential contributions can be graphically understood upon consideration of the ionwater potential of mean force (pmf) defined as

$$
W(r)=-k_{B} T \ln [g(r)],
$$

where $g(r)$ denotes the ion-oxygen radial distribution function. Figure 2 displays the pmf obtained for the $\mathrm{Na}^{+}$-water pair for the case of free dynamics (no wall included). This figure also displays the curves that result when the interactions embodied in Eqs. (1) and (2) are added to the pmf. It can be seen how the molecules within the first shell $(r$ $<3.2 \AA$ ) are effectively constrained to a well with a wall which raises steeply for increasing distance, while molecules initially beyond $r^{\ddagger}$ cannot get inside the first shell due to a 

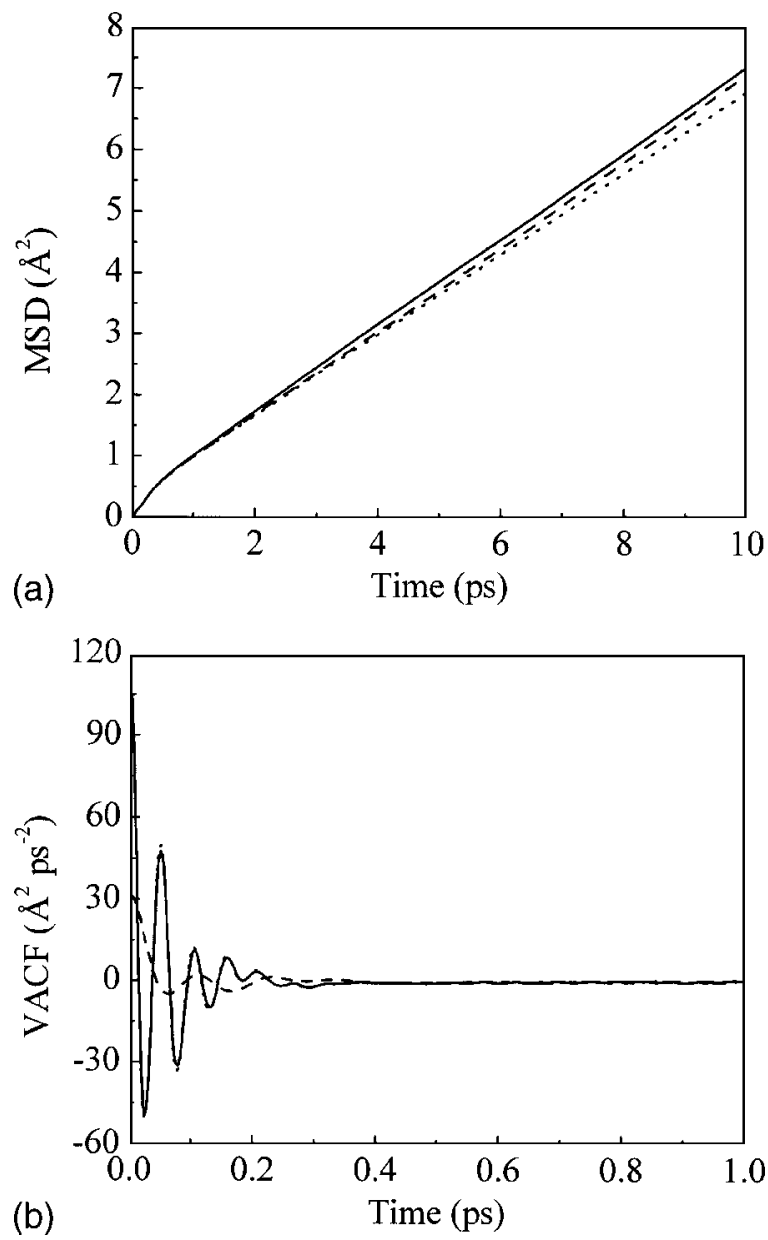

FIG. 3. $\mathrm{Li}^{+}$diffusion in ambient liquid water. Solid line, results from equilibrium simulation; dashed line, results only including portions of equilibrium run without exchanges; points, results from simulations including an effective wall between first and second hydration shells. (a) Ion mean square displacement; (b) ion velocity self-correlation function.

second barrier, which also rises steeply when the distance is reduced. The MSD and velocity tcf have been computed for a system subject to the constraints described above. The results are included in Figs. 1(a) and 1(b): the new curves are almost indistinguishable from those obtained from the subset of trajectories which do not display any exchange, confirming the previous conclusions.

While exchanges around $\mathrm{Na}^{+}$cannot be assigned to any well-defined type, ${ }^{14}$ those around $\mathrm{Li}^{+}$have in almost all cases an associative character: ${ }^{15}$ a second shell molecule enters the first shell, and after a short (variable) time a second molecule leaves. Therefore, the exchange event is characterized by a temporary hydration number larger than the mean. This behavior suggests that, contrary to the $\mathrm{Na}^{+}$case, diffusion might be slower during an exchange for $\mathrm{Li}^{+}$, considering the larger radius of the complex formed by the ion plus (five) first shell molecules. To examine this point, calculations similar to those described for $\mathrm{Na}^{+}$have been performed for $\mathrm{Li}^{+}$in ambient water. The results displayed in Figs. 3(a) and 3(b) indicate that, in line with the results for $\mathrm{Na}^{+}$, diffusion is again faster if exchanges are included $D=1.2$ in front of $D^{\prime}=1.1$ (again in units of $10^{-5} \mathrm{~cm}^{2} / \mathrm{s}$ ). Although the effect does not seem to be so marked, a $10 \%$ speed-up, it needs to be considered that exchanges are more uncommon for $\mathrm{Li}^{+}$ than for $\mathrm{Na}^{+}$: while the lifetime of a first shell molecule is 57 ps for the former ${ }^{25}$ (with four hydration molecules), it is of only 34 ps for the latter ${ }^{13}$ (with six hydration molecules). Therefore, the small difference in this case is in part due to the increased weight of trajectories which do not contain any exchange. It is worth noting in this connection that this $10 \%$ increase $\left(30 \%\right.$ in the $\mathrm{Na}^{+}$case) cannot be directly interpreted in the sense that diffusion is faster by $10 \%$ (or 30\%) during an exchange: it should be recalled that the concept of diffusion coefficient during an exchange is ill defined, given that its duration is shorter than the time required for diffusive behavior to become established.

A basic lesson to be learned, though, is that neither the increased local density during the exchanges (which in principle should hinder the ion mobility), nor the larger volume of the hydrated ion complex (which again should produce a slowdown, since, e.g., from a Stokes-Einstein point of view diffusion is slower for larger radius) are important factors. In consequence, together with the previous analysis for $\mathrm{Na}^{+}$, solvent structure disruption during the exchange seems to be the basic factor explaining the increased mobility, due to the more feeble interactions resulting from less than optimal solvent molecule orientations. Finally, comparing the results for $\mathrm{Na}^{+}$an $\mathrm{Li}^{+}$we see that certainly in the latter case, since the effect of exchanges is scarce, it is a good approximation to use a solventberg picture to understand its diffusion.

The previous results also answer one of the questions raised in the Introduction: given that the exchange times are typically longer than $10 \mathrm{ps}$ and the diffusion coefficient is well determined from a shorter portion of the MSD, it is, as we remarked there, somewhat puzzling that the solventberg picture is not mandatory in all cases. The answer is evident in Fig. 1(a) $\left(\mathrm{Na}^{+}\right.$diffusion in water), the MSD curves that correspond to trajectories with no exchanges diverge from the curve corresponding to all trajectories for times as short as 0.2 ps. The explanation is rather simple, while it is certainly true that molecules take a mean time of some 34 ps to leave the first shell, it is a key point that nothing prevents an exchange to take place immediately after a new time origin is set during the computation of the MSD. Again, Fig. 3(b), illustrates why the solventberg picture is more convenient in the case of $\mathrm{Li}^{+}$: the curves only start to (slightly) diverge for times of the order of $2 \mathrm{ps}$, when a rather good approximation of the diffusion coefficient can already be obtained.

\section{EFFECT OF DIFFUSION ON SOLVATION SHELL EXCHANGE}

We now turn to the inverse problem, summarized in the present section title. As described in the Introduction, some arguments suggest a negligible influence of the instantaneous velocity of the solute on the onset of a solvent exchange event and, actually, a thought example which supports this view was detailed there. However, a simple link is uncovered if we take upon consideration that the particle velocity and the relative velocity between the particle and a solvation molecule are correlated via simple kinematic considerations. For a solute moving inside a solvent, and assuming random directions of motion for each individual, it is evident that 
there will be a higher probability for head-on collisions with solvation molecules in front of the solute. It is important to notice that this is a probabilistic statement; collisions from behind the solute are not precluded, they are just less probable. A different way to put it is that given an instantaneous velocity for the solute, say to the right, then all molecules on the right are approaching the particle on the average (although of course some of them might be getting farther away). Note that this alternative formulation is not limited to those molecules in the immediate vicinity of the particle, molecules far away will be approaching or going away from it (for each given instantaneous particle velocity). The formulation also makes no reference to the phase of the solvent; they also apply for a low density gas situation. Despite their very general character, these simple kinematic considerations have something to say about exchange for a translating solute in a solvent. If we particularize to second shell molecules then we conclude that, for instance, those on the right of the solute will on the average get closer to the first solvation shell (with the maximum approach for those on the line of motion of the particle). Therefore, we see that purely kinetic considerations strongly condition the way in which (attempted) exchanges may start: molecules on the right side of the second solvation shell will have a higher probability of trying to enter the first solvation shell while, on the contrary, those on the left side of the first solvation shell will have a higher probability of attempting to leave (always assuming an instantaneous solute velocity to the right). The picture that results is one in which, as the solute moves in one direction, molecules in front of it try to enter the first solvation shell and molecules behind it try to exit to the second solvation shell. Of course, trying to enter or leave does not guarantee the success of the exchange but as will be shown within this is, de facto, a driving force.

\section{A. Equilibrium contribution}

The above ideas can be expressed in mathematical form assuming thermal equilibrium and a homogeneous phase so that, consequently, the formulas that result are of application to any thermal fluid. Figure 4 sketches the typical configuration in which the solute and one of the solvent molecules (of a given solvation shell) have random velocities at a given time. Taking the solute velocity direction as the origin for angles, we ask about the probability (as a function of $\theta$, see Fig. 4) that a given solvent molecule has an approaching relative velocity (which we will define as positive and negative when the two particles tend to move away). This probability is given by the expression (see the Appendix for mathematical details)

$$
\begin{aligned}
p_{+}(\theta)= & \frac{1}{\pi}\left\{\tan ^{-1}\left(\sqrt{\frac{m_{2}}{m_{1}}} \cos \theta\right)\right. \\
& \left.+\frac{1}{2} \sin \left[2 \tan ^{-1}\left(\sqrt{\frac{m_{2}}{m_{1}}} \cos \theta\right)\right]+\frac{\pi}{2}\right\},
\end{aligned}
$$

where $m_{1}$ denotes the mass of the solute, $m_{2}$ that of a solvent molecule, and the angle $\theta$ is defined over the interval $[0,180]$ deg. While we will mainly focus on this function, a parallel

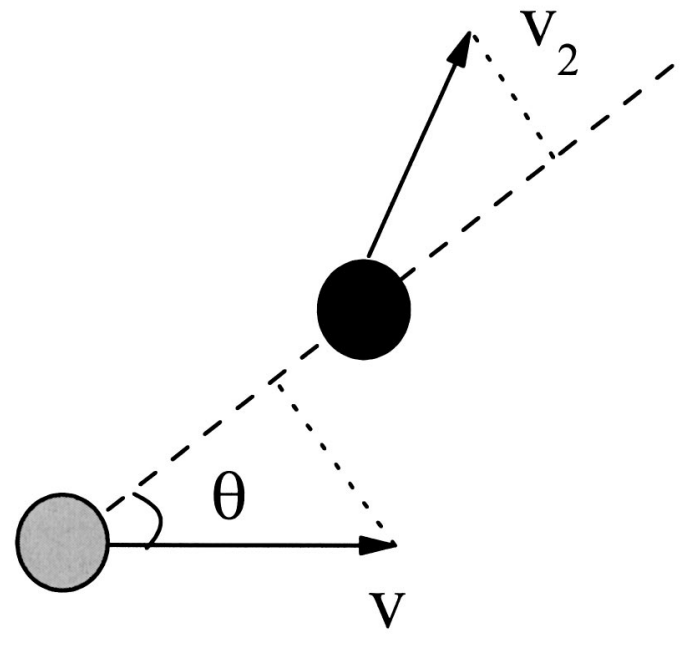

FIG. 4. Random velocities for solute (gray circle) and solvent molecule (black circle). Also shown projections of both velocities on the intermolecular axis from which $v_{r}$ [Eq. (A4)] is defined.

study can be done for the probability of having a relative velocity in the opposite direction $\left[p_{-}(\theta)\right]$, and appropriate distinctions will be made where required.

A first notable feature is the lack of dependence on temperature, which supports a similar behavior (for the attempts of solvation shell exchange) at different temperatures, as long as there exists a similar solvation structure (measured for instance by the hydration number). This constitutes an interesting aspect from a computational standpoint, since simulations of the system at higher temperatures, where exchanges are not so rare, might be a convenient starting point to gain some understanding for the behavior at lower ones (as suggested in Ref. 25), although we will not pursue this possibility here.

The mass ratio $\left(m_{2} / m_{1}\right)$ is the single parameter appearing in $p_{+}(\theta)$ and therefore it is relatively easy to understand its effect. Figure 5 displays the behavior of $p_{+}(\theta)$ for three selected values [corresponding curves for $p_{-}(\theta)$ can be constructed as a mirror image with respect to a vertical line at $\left.90^{\circ}\right]$. The wiggling curve corresponds to $m_{2} / m_{1}=1$ (equal masses for the solute and the solvent molecules), and is rep-

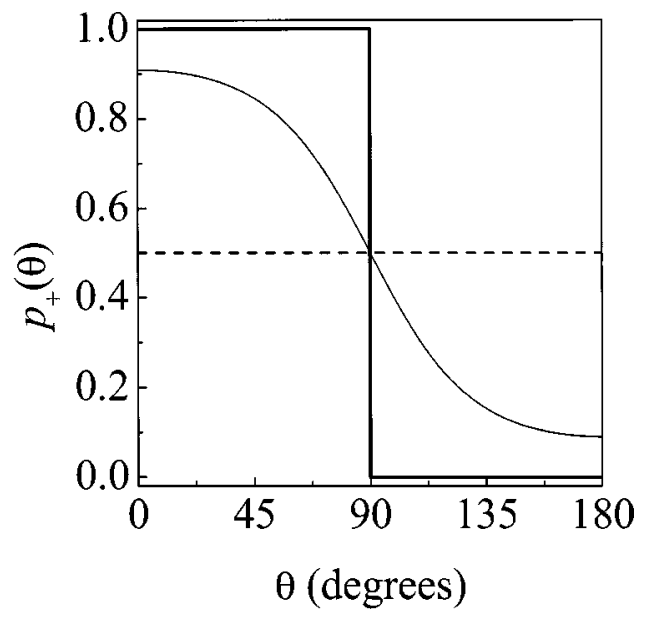

FIG. 5. $p_{+}(\theta)$, probability for a solvent molecule to approach the solute. Thick line, $m_{2} / m_{1} \rightarrow \infty$; dashed line, $m_{2} / m_{1} \rightarrow 0$; thin line, $m_{2} / m_{1}=1$. 
resentative of the general situation. It illustrates how the probability of having an approaching relative velocity is larger at $\theta=0^{\circ}$ and attains its lowest value at $\theta=180^{\circ}$, effectively resulting in an asymmetric distribution around the instantaneous solute velocity, which constitutes a central result of this work. Two interesting limiting cases are also included. For $m_{2} / m_{1} \rightarrow 0$ (particle mass much larger than that of a solvent molecule) we recover the limiting case that was qualitatively discussed in the Introduction (with no real diffusion). We see how the probability distribution is flat $\left[p_{+}(\theta)=1 / 2\right]$ : all molecules around the central massive particle have an equal (symmetric) probability of attempting to get into its vicinity (and similarly for the probability of attempting to escape). The second limiting case corresponds to $m_{2} / m_{1} \rightarrow \infty$, a very light solute in comparison with massive (and slow) solvent molecules. Here we obtain the step function depicted in Fig. 5, defined by

$$
p_{+}(\theta)= \begin{cases}1 & \text { for } 0 \leqslant \theta<90^{\circ} \\ 0 & \text { for } 90^{\circ}<\theta \leqslant 180^{\circ},\end{cases}
$$

which is easily interpreted: the solute undergoes a random motion in a maze of static scatterers, which will never "chase" it and therefore no approaching molecules can be found for any angle greater than $90^{\circ}$ [the corresponding inverted behavior would be found for $\left.p_{-}(\theta)\right]$.

Finally, it should be noted that the function just analyzed corresponds to the probability for any given molecule to have an approaching relative velocity with respect to the solute. A related, but different, function is the fraction of molecules found for each angle of all those that have inward velocity [which will be denoted $\wp_{+}(\theta)$ ]. Given that there is cylindrical symmetry around the solute velocity, more molecules are found for angles close to $90^{\circ}$ than for smaller (close to $0^{\circ}$ ) or larger angles (close to $180^{\circ}$ ). On the contrary, $p_{+}(\theta)$, as we have seen before, has a maximum at $\theta=0^{\circ}$. In consequence, the maximum of $\wp_{+}(\theta)$ will be found somewhere between $0^{\circ}$ and $90^{\circ}$. The result is (see the Appendix for details)

$$
\begin{aligned}
\wp_{+}(\theta)= & \frac{\sin (\theta)}{\pi}\left\{\tan ^{-1}\left(\sqrt{\frac{m_{2}}{m_{1}}} \cos \theta\right)\right. \\
& \left.+\frac{1}{2} \sin \left[2 \tan ^{-1}\left(\sqrt{\frac{m_{2}}{m_{1}}} \cos \theta\right)\right]+\frac{\pi}{2}\right\},
\end{aligned}
$$

where the only difference with $p_{+}(\theta)$ is the sine function preceding the braces.

Figure 6 displays the function $\wp_{+}(\theta)$ for each of the three examples that were just discussed above in terms of $p_{+}(\theta)$. For $m_{2} / m_{1} \rightarrow 0$ the distribution displays a maximum at $90^{\circ}$, showing that $\wp_{+}(\theta)$ can be somewhat misleading about the process: although all molecules (for any angle) have the same probability of having an approaching velocity (in this particular limit), collecting all the molecules for a given angle results in an apparent nonsymmetric distribution of molecules trying to enter. With this cautionary note in mind, we see how in the opposite limit $\left(m_{2} / m_{1} \rightarrow \infty\right)$ the distribution also peaks at $90^{\circ}$. Finally, all the intermediate cases are characterized by a maximum at an angle lower than $90^{\circ}$,

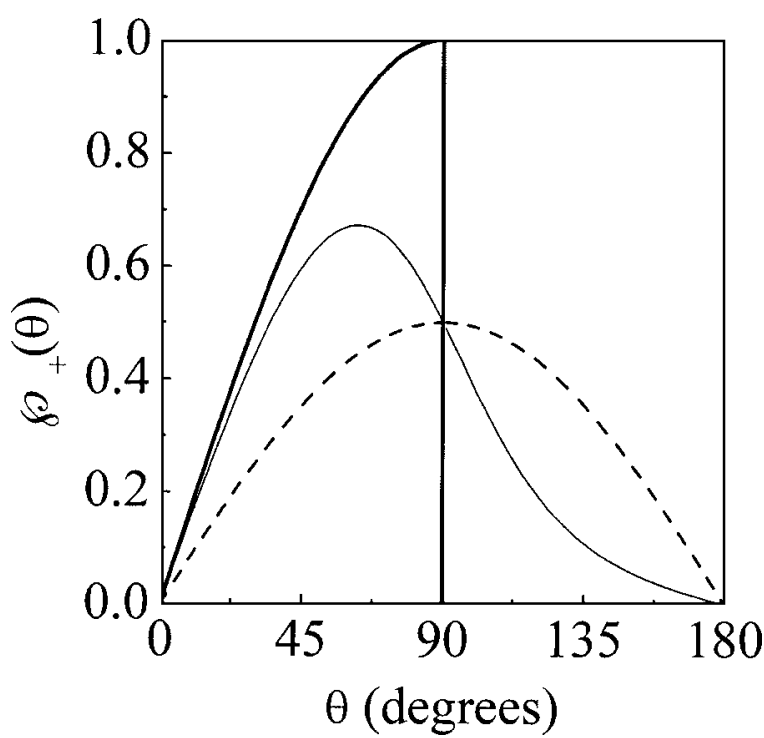

FIG. 6. $\wp_{+}(\theta)$, fraction of molecules having an inward velocity for each $\theta$. Thick line, $m_{2} / m_{1} \rightarrow \infty$; dashed line, $m_{2} / m_{1} \rightarrow 0$; thin line, $m_{2} / m_{1}=1$.

which reflects that if the attempted exchanges are monitored and collected as function of $\theta$, a maximum at this angle will be found, and not along the velocity direction [as might in principle be expected from $\left.p_{+}(\theta)\right]$.

Given the probabilistic nature of the formulas just derived, it is obvious that by themselves they cannot predict when and how an exchange event will start, they should be regarded instead as a sort of "sum rule." As such, they can help interpret averaged results for the stereochemistry of the exchanges. We take the case of the hydration shell exchange process around the lithium ion in ambient water as an illustrative example. This system, which has been recently studied, ${ }^{15}$ is characterized by a tetrahedral equilibrium solvation structure and a substantial variety of distinct exchange classes. A priori arguments would suggest that when the exchange is simultaneous (one molecule entering the first shell while another leaves) the mechanism should have a trans character. This hypothesis is based on the well-known $\mathrm{S}_{\mathrm{N}} 2$ reaction mechanism (Walden inversion), in which the attacking and leaving groups form $180^{\circ}$. But in contradiction with this hypothesis, it is found ${ }^{15}$ that the number of cis exchanges is more than half of those assigned to trans exchanges. While a satisfactory explanation could not be given in Ref. 15, it now seems clear that this finding is due to the kinetic constraints just described. As we have seen, $\wp_{+}(\theta)$ peaks at an angle of $\approx 60^{\circ}$ (applying formula 6 to the pair $\left.\mathrm{Li}^{+}+\mathrm{H}_{2} \mathrm{O}\right)$, while $\wp_{-}(\theta)$ would have a corresponding maximum at a value of $120^{\circ}$. This implies that the angle between the entering and leaving water molecules may span the range from $60^{\circ}\left(=120^{\circ}-60^{\circ}\right.$, assuming a null dihedral angle between the plane defined by the ion velocity and the leaving molecule velocity, and the plane formed by the ion velocity and the entering molecule velocity) up to $180^{\circ}$ (for a dihedral angle of $180^{\circ}$ ). This broad range of possibilities results assuming the most probable entrance and exit angles, and thus is further reinforced if we consider the broad dispersion of angles apparent in Fig. 6. Therefore, we see that cis exchanges are not precluded at all and that, instead, a con- 
tinuum between cis and trans is most probably the general rule. In short, the substantial number of cis exchanges found in the simulations is due to the fact that more molecules are available at angles larger than $0^{\circ}$. Actually, the considerations above suggest that a classification in terms of cis and trans exchange classes is a concept which should not be pursued for exchange processes, at least for the types of exchanges considered here. ${ }^{26}$ Finally, as explained above, if the exchanges would be normalized by the number of water molecules available at each angle, then the distribution $p_{+}(\theta)$ is obtained for the entering molecules and $p_{-}(\theta)$ for those leaving (which peak, respectively, at $0^{\circ}$ and $180^{\circ}$ ): in consequence, the most probable exchange would have a trans character as initially expected.

\section{B. Nonequilibrium contribution}

The previous discussion provides an "equilibrium" estimation for the exchanges, in the sense that we have computed the probability that a given second shell solvent molecule might attempt to enter the first shell $\left[p_{+}(\theta)\right]$, or the fraction of molecules trying to enter for a given angle $\left[\wp_{+}(\theta)\right]$. However, we now have to consider nonequilibrium effects, meaning that the attempted exchange will fail or succeed depending on the detailed dynamics of the system. In the end, the success or failure depends on the combination of equilibrium (probability of attempting an exchange) and nonequilibrium contributions (dynamics starting at the attempted exchange). A pessimistic view would anticipate that the nonsymmetric distribution just discussed might get blurred by this additional contribution so that, finally, no noticeable correlation will be found between (successful) exchanges and the instantaneous solute velocity. However, there are strong arguments in the opposite direction stemming from reaction rate theory. The problem of solvation shell exchange, particularly in the vicinity of an ion, was first tackled from the standpoint of activated reactive process for the $\mathrm{Na}^{+}$-water system. ${ }^{13}$ There it was shown that the exchange rate (note that this is a quantity averaged over all angles) can be expressed as the product of equilibrium contributions [determined from transition state theory (TST)] and a nonequilibrium contribution dependent on the dynamics (transmission coefficient $\kappa$ ). This approach has been subsequently applied to the aforementioned case of $\mathrm{Li}^{+}(\mathrm{aq})$ in a broad range of thermodynamic conditions ${ }^{25}$ and, at a more detailed level, in ambient water. ${ }^{15}$ A first lesson from this work is that TST provides an acceptable estimation of the exchange rate, predicting at least the right order of magnitude, which is remarkable if we consider that exchange rates span more than 15 orders of magnitude. ${ }^{27}$ TST assumes, as applied to the present case, that all molecules crossing the transition state (basically the division between first and second solvation shells) with inward velocity will finally end up in the first shell. We must conclude that (in this approximation) $p_{+}(\theta)$ [or $\wp_{+}(\theta)$ ] represents not only the probability of attempted exchanges but the real distribution of successful exchanges and, therefore, all exchanges (for any sort of solute or solvent) obey exactly the same rules.

It must be said though that the transmission coefficient is rather low in the cases that have been studied so far, with values ranging from $\approx 0.4$ (for $\mathrm{Li}^{+}$in supercritical water ${ }^{25}$ ) down to 0.14 (for $\mathrm{Li}^{+}$in ambient water ${ }^{15}$ ), so that its effect certainly has to be considered. However, this correction will affect the estimated distribution of successful exchanges $\left[p_{+}(\theta)\right.$ or $\left.\wp_{+}(\theta)\right]$ only if a sort of angle-dependent transmission coefficient is found, so that the probability for an attempt of being successful would depend on the angle $\theta$. The only way to ascertain this point seems to be a case by case analysis, performed by MD simulation of the systems. In principle, this could be done but it is a computationally difficult task, for example, the computation has to be performed separately for each angle, so that it has to be lengthened proportionally to the number of intervals in which the interval $\left[0^{\circ}, 180^{\circ}\right]$ is divided (for a given statistical tolerance). With these considerations in mind we have chosen three examples where this study is still feasible.

In each case we have computed the previously defined function $\wp_{+}(\theta)$ (the fraction of molecules found for each angle of all those with inward velocity), which as previously explained takes into account the equilibrium effects. To scrutinize the nonequilibrium effects we have also computed the fraction of molecules found for each angle of all those that finally become stabilized within the first shell [denoted as $\left.\wp_{+}^{\text {delay }}(\theta)\right]$. It is important to note that $\wp_{+}^{\text {delay }}$ is not the fraction of successful molecules from all those that tried to enter initially. If defined that way, because of the strong reduction embodied in $\kappa$ (transmission coefficient), $\wp_{+}^{\text {delay }}(\theta)$ would be very low for any angle and not easily comparable with $\wp_{+}(\theta)$. With these definitions, the basic idea is that, if $\kappa$ is not dependent on $\theta$, then we should obtain $\wp_{+}(\theta)=\wp_{+}^{\text {delay }}(\theta)$, i.e., all the molecules trying to enter have the same chance of being successful irrespective of the attack angle $\theta$. Any dependence of $\kappa$ on $\theta$ will show up as a difference between $\wp_{+}(\theta)$ and $\wp_{+}^{\text {delay }}(\theta)$.

First, we have addressed $\mathrm{Na}^{+}$in ambient water, a case characterized by a low transmission coefficient $(\approx 0.21)$ and a first solvation shell of six molecules. ${ }^{13}$ A long run of $14 \mathrm{~ns}$ has been performed at $298 \mathrm{~K}$, during which $\approx 10000$ attempts by second shell water molecules to enter the first shell have been recorded. The angle between the relative velocity and the instantaneous ion velocity has been computed in each case, which allows us to estimate $\wp_{+}(\theta)$. As it can be seen in Fig. 7(a), this numerical estimation nicely matches the theoretical prediction of formula (4). In order to ascertain the nonequilibrium contribution, each of these attempts has been followed during $1.5 \mathrm{ps}$. This time is chosen in accordance with the reactive flux function computed in Ref. 13, where it was shown that after $\approx 0.5 \mathrm{ps}$ it levels off and reaches a plateau, from which its mean value can be identified with the aforementioned transmission coefficient $(\kappa$ $=0.21$ ). It has been found that, consistently with the latter value (which can also be interpreted as the ratio of successful exchanges over total number of attempts), a total of $\approx 2100$ second shell molecules become stabilized within the first solvation shell. If these successful attempts are assigned to the corresponding angle (with an angle interval of $1^{\circ}$ ), it results in the curve depicted in Fig. 7(b). Despite the substantial amount of noise, it is rather clear that this curve is rather 

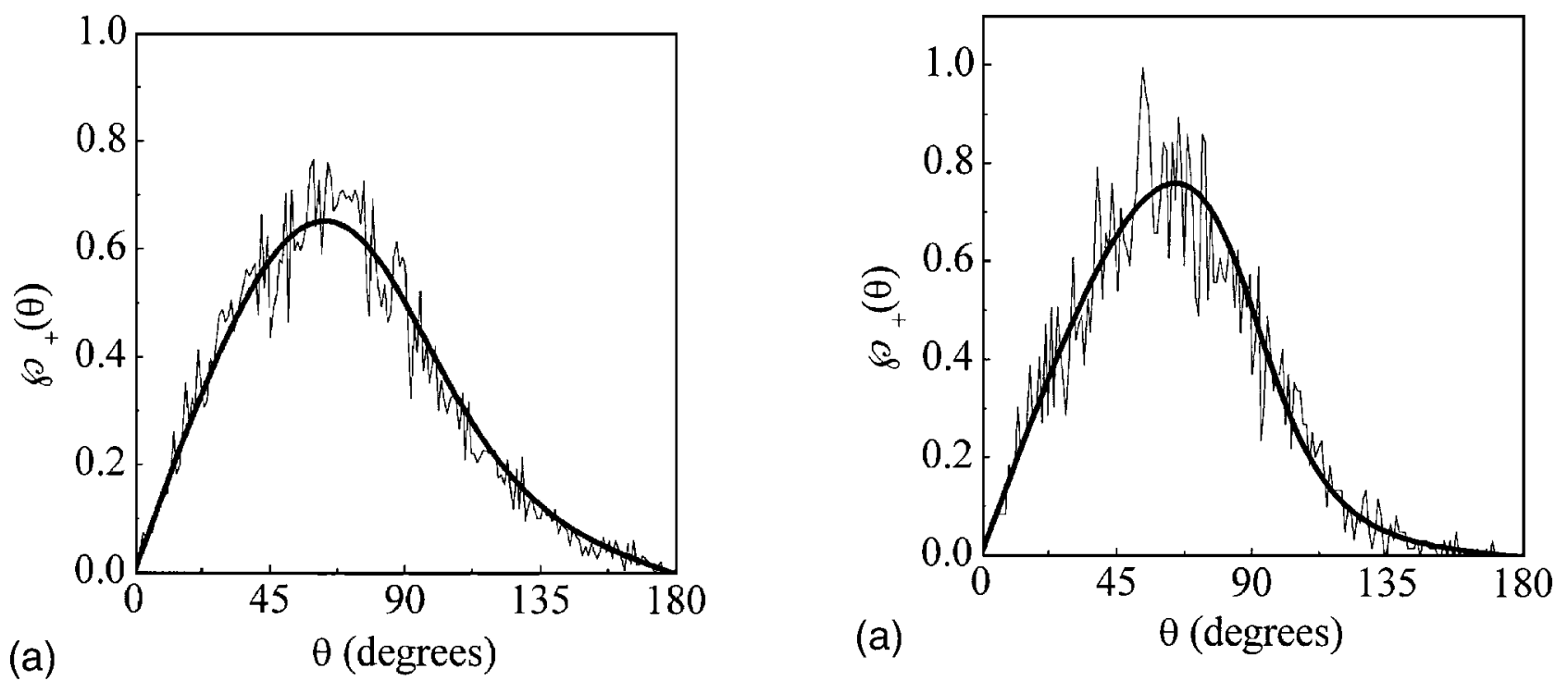

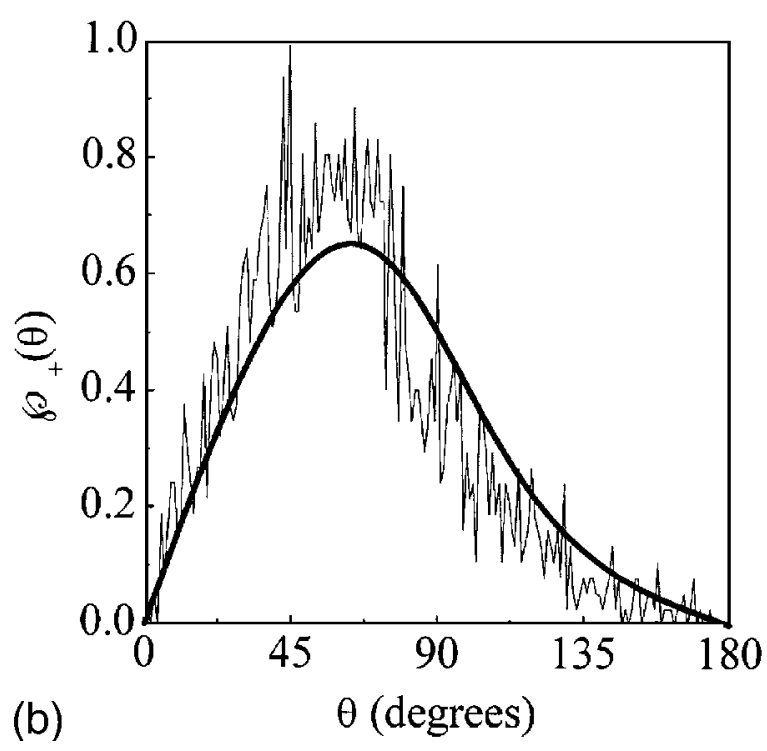

FIG. 7. (a) Thick line, theoretical $\wp_{+}(\theta)$ for $\mathrm{Na}^{+}$in ambient liquid water; thin line, computed from MD simulation; (b) thin line, computed $\wp_{+}^{\text {delay }}(\theta)$ for $\mathrm{Na}^{+}$in ambient liquid water; thick line, theoretical curve for $\wp_{+}(\theta)$.

similar to the theoretical curve for attempted exchanges, although it seems slightly higher at small angles and closer to zero at larger angles. In conclusion, the probability of attempted exchanges is, de facto, the one that conditions the distribution of successful exchanges, with a possible small correction from the nonequilibrium contribution.

$\mathrm{Li}^{+}$in supercritical water is the second case that has been analyzed numerically. While in ambient water the transmission coefficient seems to be rather low for the systems analyzed so far, ${ }^{13,15,25}$ it has been found that in supercritical water it can exceed by $0.4 .^{25}$ Together with the lower number of hydration molecules (four), these constitute interesting differences with the preceding case. Simulation runs of $6 \mathrm{~ns}$ were performed during which $\sim 4000$ trajectories of water molecules entering the first solvation shell were followed (for the simulations of $\mathrm{Li}^{+}$in supercritical water we have used the same ion-water interaction parameters as in Ref. 25, where a detailed study of lithium diffusion in this phase was

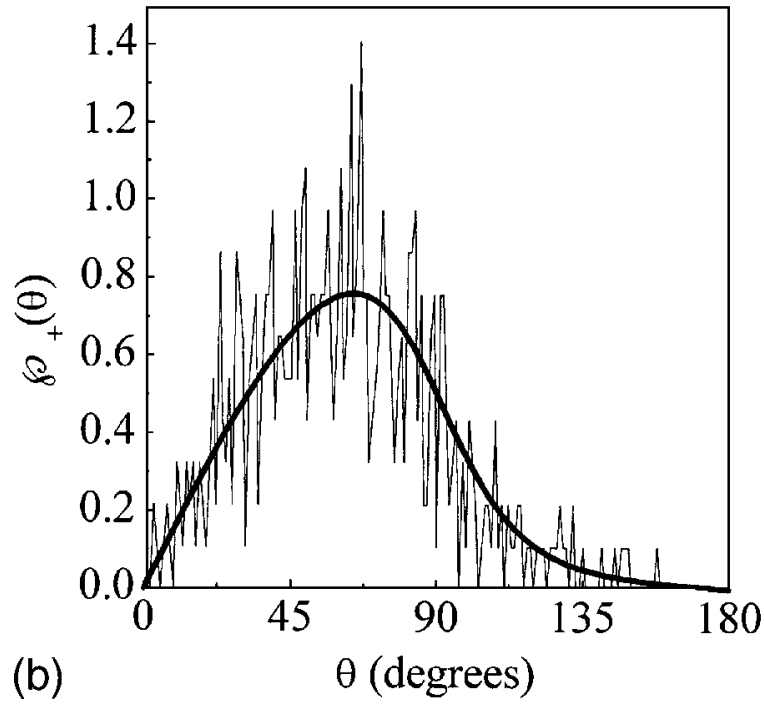

FIG. 8. (a) Thick line, theoretical $\wp_{+}(\theta)$ for $\mathrm{Li}^{+}$in supercritical water $(T$ $=683 \mathrm{~K}, \rho=0.20 \mathrm{~g} \mathrm{~cm}^{-3}$ ); thin line, computed from MD simulation; (b) thin line, computed $\wp_{+}^{\text {delay }}(\theta)$ for $\mathrm{Li}^{+}$in supercritical water; thick line, theoretical curve for $\wp_{+}(\theta)$. Similar results are found at $\rho=0.31 \mathrm{~g} \mathrm{~cm}^{3}$ and $\rho$ $=0.48 \mathrm{~g} \mathrm{~cm}^{-3}$.

performed, and the SPC/E model for water ${ }^{28}$ ). Three different densities were chosen at the temperature of $683 \mathrm{~K}: 0.20$, 0.31 , and $0.48 \mathrm{~g} \mathrm{~cm}^{-3}$. The time interval over which the trajectories are followed before deciding if the attempt is successful has been chosen as in the previous case. Figures 8(a) and 8 (b) display the corresponding results. Again, the results are rather similar to the case of $\mathrm{Na}^{+}$in ambient water.

Despite their differences, the previous examples are both representative of ionic diffusion (albeit in different phases), sharing some important common characteristics such as low hydration number and strong interactions. Since the formulas have general applicability, it is of interest to examine systems that differ markedly as far as these aspects are concerned. We have addressed a neat simple liquid, the much studied liquid argon, ${ }^{23,24,29,30}$ which has the following interesting characteristics: a solvation number substantially larger $(\approx 12)$ and almost no free energy barriers to exchange (see below). Furthermore, it constitutes a computationally convenient system: 
it is easier to obtain good statistics since now all the atoms can be used, instead of a single ion as before. The system is composed of $500 \mathrm{Ar}$ atoms at a temperature of $112 \mathrm{~K}$ and a density of $1.479 \mathrm{~g} \mathrm{~cm}^{-3}$. We have performed an analysis on the solvation shell exchange process.

First, from the radial distribution function we find that the barrier to exchange, viewed in a unimolecular dissociation perspective (as done for ions in water ${ }^{13,15,25}$ ), is $\approx 1.6 k_{B} T$ [computed from the free energy defined in Eq. (3)]. This low barrier shows that the process is not well described as an activated one, even though we have chosen a state point where the barrier is probably (close to) the highest possible in liquid argon. ${ }^{29}$ Since the velocity time correlation function is known to decay in less than 1 ps (comparable to the case of an ion in liquid water and to the corresponding reactive flux function), we have also followed the incoming atoms during $1.5 \mathrm{ps}$ before checking if they end up in the first shell. The results, at each state point, correspond to equilibration runs of 50000 steps (where the velocities were rescaled to get the reference temperature of the simulation) followed by production runs of 1000000 steps. Figures 9(a) and 9(b) display the corresponding results. Again, there is a perfect match with the theoretical curve for $\wp_{+}(\theta)$ [Fig. 9(a)]. Thanks to the better statistical sampling the deviation of the distribution of successful exchanges $\left[\wp_{+}^{\text {delay }}(\theta)\right]$ from the theoretical curve is now perfectly discernible (Fig. 9), and confirms the deviation hinted in the plots corresponding to $\mathrm{Na}^{+}$ and $\mathrm{Li}^{+}$. We see that head-on collisions (small angles) have a (slightly) higher probability of being successful [values above $\wp_{+}(\theta)$ ] in comparison with those at large angles [curve closer to zero than $\left.\wp_{+}(\theta)\right]$. A simple explanation suffices to account for this: the mean (approaching) relative velocity is larger at small angles rather than at angles close to $180^{\circ}$ (another reflection of the fact that solvent molecules at small angles are approaching the solute, on the average, and those at large angles are departing from it, on the average). This higher velocity should facilitate the success of the exchange at small angles, and this is what is actually found for liquid argon, and is hinted at in the plots for the cations in water. Evidently, the nonequilibrium contribution slightly enhances the difference between entering and leaving angles.

\section{Effect on the exchange rate}

The previous sections have addressed the effect of the instantaneous diffusive state of the solute on the mechanism of the exchange process. It has been shown that a symmetric distribution of entering (and leaving) solvent molecules for a static solute turns into an asymmetric one if the solute is allowed to diffuse. Since exchanges occur in both cases, we are led to also ask whether the kinetics (exchange time) is affected as well when the solute mass is increased or decreased (without altering the force field). It will be shown that this effect actually exists and can be understood from purely equilibrium considerations. To illustrate this point we have extended our simulations for $\mathrm{Li}^{+}$by artificially increasing its mass so that, everything else unchanged, the lithium ion is effectively static. In particular, we have taken $\mathrm{Li}^{+}$in water at $T=683 \mathrm{~K}$ and $\rho=0.2 \mathrm{~g} \mathrm{~cm}^{-3}$. We adopt the usual
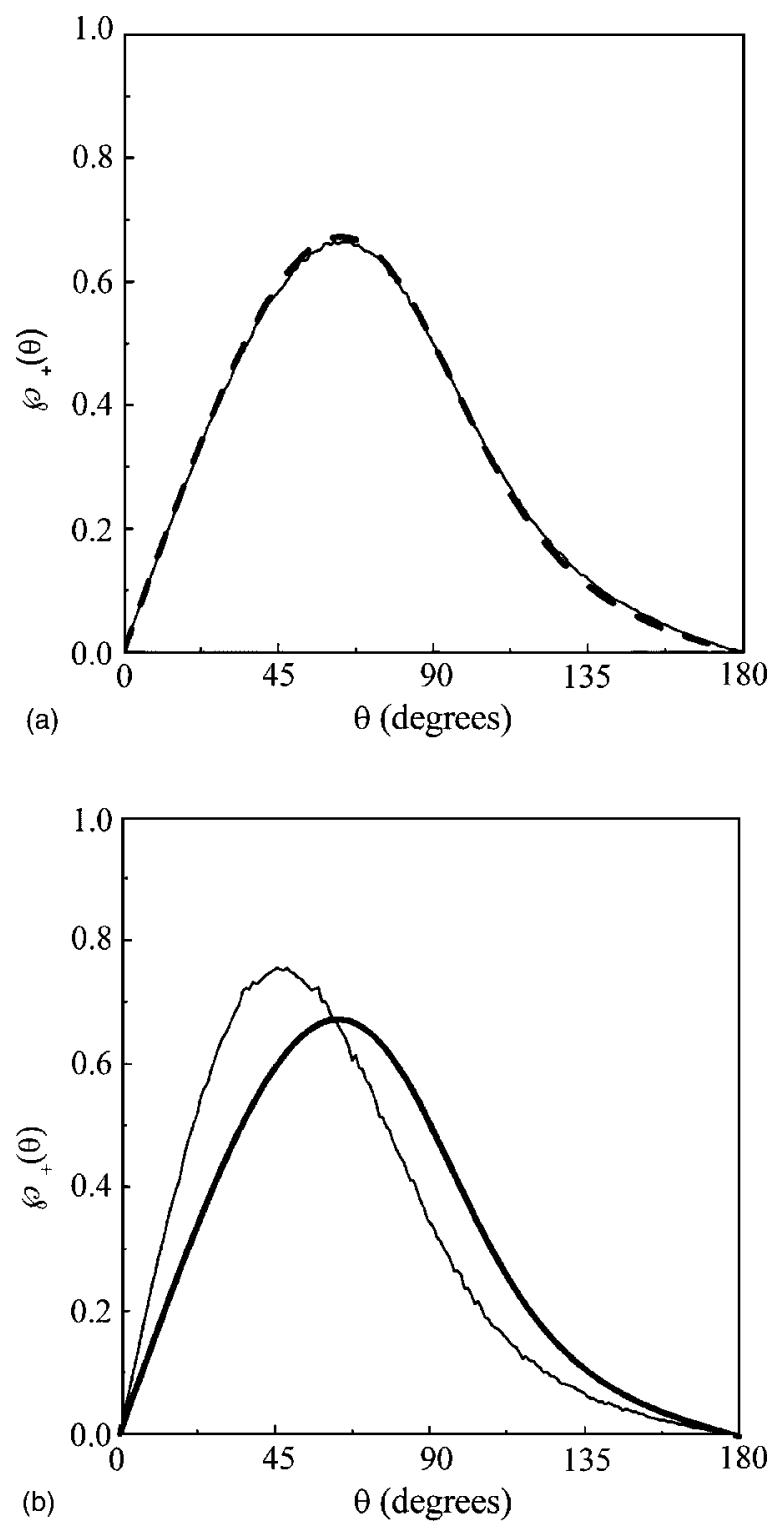

FIG. 9. (a) Thick dashed line, theoretical $\wp_{+}(\theta)$ for liquid $\operatorname{Ar}(T=112 \mathrm{~K}, \rho$ $=1.479 \mathrm{~g} \mathrm{~cm}^{-3}$ ); thin line, computed from MD simulation; (b) thin line, computed $\wp_{+}^{\text {delay }}(\theta)$ for liquid Ar, thick line, theoretical curve for $\wp_{+}(\theta)$.

definition of exchange time $e^{9,13,25}$ as the time constant of the exponential fit to the survival function defined

$$
n(t)=\frac{1}{N_{h}} \sum_{i=1}^{N_{h}} \theta_{i}(r, t) \theta_{i}(r, 0),
$$

where $\theta(r, t)$ is 1 if the molecule is within the first hydration shell (defined by a maximum separation $r^{\ddagger}$ between the ion and the water molecule center of mass), and 0 otherwise. $N_{h}$ denotes the number of water molecules initially within the first shell, and a molecule is considered to have left the first shell only if it has been out for more than $\tau^{*}=2$ ps.

Obviously, we now find that for an ion of infinite mass the distribution of exchanges is symmetric and, what is of interest here, that the exchange time has a value of $\tau_{\infty}$ $=10.8 \mathrm{ps}$, to be compared with a faster exchange time of $\tau$ $=6.9 \mathrm{ps}$ if the lithium ion is assigned its correct mass, ${ }^{25}$ so that a ratio of $\tau / \tau_{\infty}=0.6$ results. To understand the origin of 
this effect we will adopt the approach that has been cursorily described within the previous sections, namely, to view the exchange as an association-dissociation process. ${ }^{13,15,25}$ From this standpoint the exchange rate is written, following the usual reaction rate theory formulas, as a product $k=k^{\mathrm{TST}} \kappa$, where $k^{\mathrm{TST}}$ is obtained from

$$
k^{\mathrm{TST}}=\sqrt{\frac{k_{B} T}{2 \pi \mu}} \frac{\left(r^{\ddagger}\right)^{2} e^{-\beta W\left(r^{\ddagger}\right)}}{\int_{0}^{r^{\ddagger}} d r r^{2} e^{-\beta W(r)}},
$$

where $W(r)$ has been defined in Eq. (3).

Equation (8) shows that the equilibrium contribution $k^{\mathrm{TST}}$ depends on the square root of the inverse reduced mass, the only varying parameter since $W(r)$ will remain unchanged even if the masses are varied (as the force field has not been altered). If an infinite mass is assigned to the lithium ion, we will have $\mu_{\infty}=m_{\text {water }}$, i.e., the reduced mass is that of the water molecule, whereas for real masses we will have $\mu<\mu_{\infty}$. Therefore, together with Eq. (8) and assuming that $\kappa$ is the same in both cases, we have the following theoretical (equilibrium) estimation for the ratio of exchange times:

$$
\frac{\tau}{\tau_{\infty}}=\frac{k_{\infty}^{\mathrm{TST}}}{k^{\mathrm{TST}}}=\sqrt{\frac{\mu}{m_{\text {water }}}}=0.5,
$$

rather close to the previous value of 0.6 (obtained from direct simulation). Given the indeterminacy in the fits of the survival function, we conclude that the fundamental explanation lies in the variation of the reduced mass: exchanges are slower if the solute mass is increased due to the concomitant larger reduced mass of the pair, with a possibly null effect of the dynamic correction $\kappa$. Indeed, the present example constitutes an extreme case as far as ionic diffusion in water is concerned; for more massive ions the difference between $\mu$ and $\mu_{\infty}$ is smaller and therefore the ratio will become closer to unity (as it is obvious that the larger the ion mass, the closer we are to the static solute limit). Finally, it is important to note how useful the association-dissociation perspective has been in order to easily understand this particular issue. Although the existence of a more optimal (i.e., with a higher $\kappa$ ) reaction coordinate for the exchange process would be of interest, it has been just shown that the ion-water distance results in simple analytical formulas [Eq. (8)] which provide useful (quantitative) understanding on the trends.

\section{CONCLUSIONS}

The link between exchange events and the diffusive process has been studied from two different perspectives. First, two independent methods have been devised to quantitatively estimate the commonly accepted diffusion speed-up induced by exchanges. This methodology has been applied to $\mathrm{Li}^{+}$and $\mathrm{Na}^{+}$in liquid water, finding that diffusion increases by $10 \%$ and $30 \%$, respectively. The former case is particularly interesting: although exchanges are characterized by larger than average hydration shells (associative process), mobility is still faster. Therefore, it is reasonable to infer that solvent structure disruption during the exchange, with its more feeble concomitant interactions, is a key factor explaining the increased mobility. Moreover, it shows that neither the increased local density nor the larger volume of the hydrated ion complex (in principle relevant from a Stokes theory point of view) are decisive factors for this particular issue, as they would both predict a mobility slowdown. In this work only the $\mathrm{Li}^{+}$and $\mathrm{Na}^{+}$cations have been addressed, as for these cases the kinetics and dynamics of the exchange have been studied in detail. ${ }^{13-15,25}$ It would be interesting to extend the present study to anions, ${ }^{6,10,31-33}$ especially in water where hydrogen bonding effects could introduce new features. In this connection it is important to note that a proper modeling of the hydrogen bonds for anions most probably requires the inclusion of polarizability, ${ }^{33}$ as first shell structure seems to be critically dependent on this effect. An assessment of the different methods available to this end is currently underway. ${ }^{34}$

The influence of the instantaneous diffusive state of the solute on the exchange properties has constituted the second main focus of interest in this work. It has been shown that purely kinematic considerations, together with the assumption of thermal equilibrium, are sufficient to derive analytic laws for the probability of entrance to the first solvation shell and, similarly for that of escape. These expressions are of general applicability and imply that, as a rule of thumb, the majority of the solvent molecules will be found entering at an angle of some $60^{\circ}$ with respect to the instantaneous solute velocity, while those leaving will be mostly found forming an angle of some $120^{\circ}$. Despite the generality and purely kinematic character of these considerations, they have shown to be extremely useful in characterizing the results for the nontrivial cation in water systems, as well as for liquid argon (as noted above, the case of anions in water remains to be investigated). Furthermore, it has been shown that dynamical corrections to these expressions, while being of secondary importance, tend to slightly increase the difference between entrance and exit angles, with the former becoming closer to $0^{\circ}$ (and to $180^{\circ}$ the latter). Finally, it has been argued that the distinction between cis and trans exchanges, for exchanges involving the instantaneous exchange of two molecules, is probably not possible for simple ionic exchange processes.

\section{ACKNOWLEDGMENTS}

This work was supported by EC TMR network under Grant No. HPRN-CT-2000-19 (Solvation Dynamics and Ionic Mobility in Conventional and Polymer Solvents), the SNF, MCYT project BFM2001-2077, the CNRS, and U.S. NSF Grants Nos. CHE-0108314 and CHE-0417570.

\section{APPENDIX}

We first derive the probability $\left(p_{+}\right)$for a solvent molecule to have an approaching relative velocity with the solute. According to the generic configuration depicted in Fig. 4 , the solute velocity defines the origin for angles. The modulus of this velocity will have the (normalized) probability density 


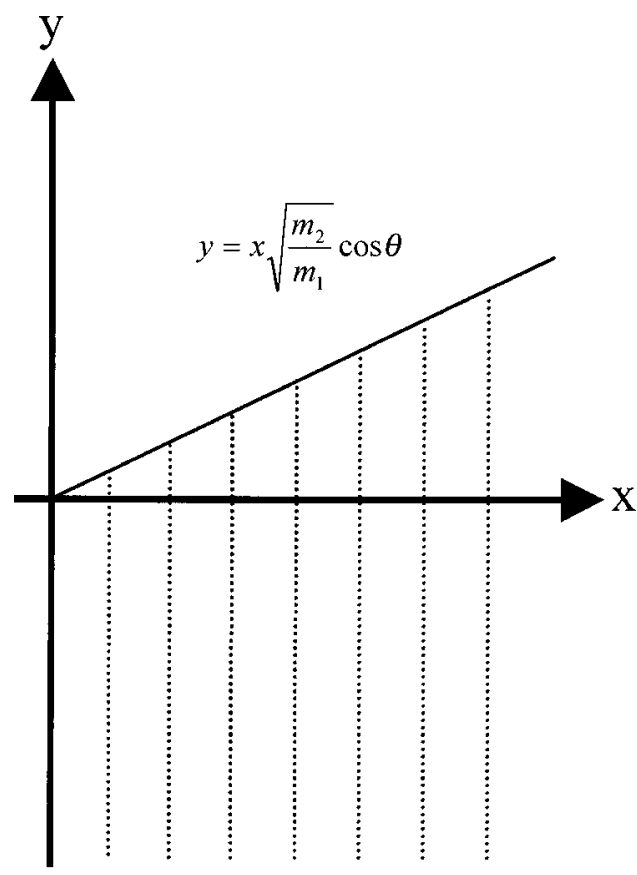

FIG. 10. Domain of integration indicated by shaded area.

$$
p(v)=\frac{4}{\sqrt{\pi}}\left(\frac{m_{1}}{2 k_{B} T}\right)^{3 / 2} v^{2} e^{-\left(m_{1} v^{2}\right) / 2 k_{B} T}
$$

where $m_{1}$ denotes the solute mass, and the function is defined over $[0, \infty]$.

The velocity distribution function for the solvent molecule velocity along the axis joining the solute and the solvent molecule is given by

$$
p\left(v_{2}\right)=\sqrt{\frac{m_{2}}{2 \pi k_{B} T}} e^{-\left(m_{2} v_{2}^{2}\right) / 2 k_{B} T},
$$

where $m_{2}$ is the mass of a solvent molecule. It should be noted that, in contrast with the previous case, now this function is defined over the interval $[-\infty, \infty]$.

It follows from the previous considerations that the probability density that the modulus of the solute velocity is $v$ and the solvent molecule has a velocity $v_{2}$ along the line joining both particles is

$$
\begin{aligned}
p\left(v, v_{2}\right) & =p(v) p\left(v_{2}\right) \\
& =\frac{m_{1} \sqrt{m_{1} m_{2}}}{\pi\left(k_{B} T\right)^{2}} v^{2} e^{-\left(m_{1} v^{2}\right) / 2 k_{B} T} e^{-\left(m_{2} v_{2}^{2}\right) / 2 k_{B} T} .
\end{aligned}
$$

A simple projection of the modulus of the solute velocity (see Fig. 4) on the intermolecular axis shows that the relative velocity is given by

$$
v_{r}=v \cos \theta-v_{2}
$$

the difference defined so that, when both particles approach each other, $v_{r}$ is positive.

Finally, the probability we are seeking can be found integrating the two-dimensional distribution $p\left(v, v_{2}\right)$ [Eq. (A3)] under the constraint $v_{r}>0$, i.e.,

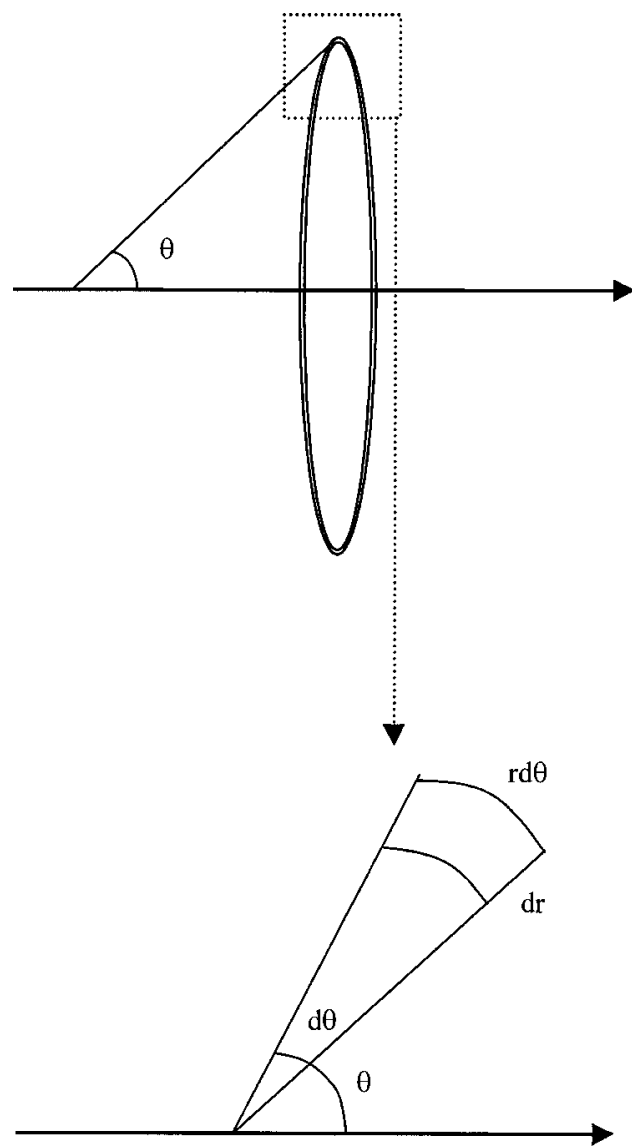

FIG. 11. Sketch of the volume of the ring at angle $\theta$

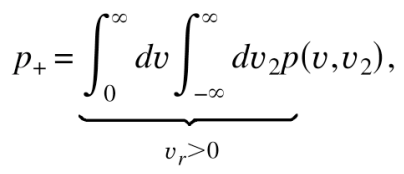

Changing variables $\left[x \equiv v\left(m_{1} / 2 k_{B} T\right)^{1 / 2}, \quad y \equiv v_{2}\left(m_{2} /\right.\right.$ $\left.2 k_{B} T\right)^{1 / 2}$, Eq. (A5) can be rewritten as

$$
p_{+}=\frac{4}{\pi} \underbrace{\int_{0}^{\infty} d x \int_{-\infty}^{\infty} d y x^{2} e^{-x^{2}-y^{2}}}_{\cos \theta \sqrt{\frac{m_{2}}{m_{1}}} x-y>0}
$$

The domain of integration is depicted in Fig. 10, which suggests a change to polar coordinates $(x \equiv r \cos \phi$, $y \equiv r \sin \phi)$. After this change the 2D integral is separable

$$
p_{+}=\frac{4}{\pi} \int_{0}^{\infty} d r r^{3} e^{-r^{2}} \int_{-\pi / 2}^{\arctan \left(\sqrt{\left(m_{2} / m_{1}\right)} \cos \theta\right)} d \phi \cos ^{2} \phi,
$$

both integrals are trivially done, and the final result is

$$
\begin{aligned}
p_{+}(\theta)= & \frac{1}{\pi}\left\{\tan ^{-1}\left(\sqrt{\frac{m_{2}}{m_{1}}} \cos \theta\right)\right. \\
& \left.+\frac{1}{2} \sin \left[2 \tan ^{-1}\left(\sqrt{\frac{m_{2}}{m_{1}}} \cos \theta\right)\right]+\frac{\pi}{2}\right\} .
\end{aligned}
$$

We see how the result automatically contains a dependence on $\theta$. As a first trivial check, in the limit case $m_{2} / m_{1} \rightarrow 0$ the result is $p_{+}=1 / 2$, that is, all molecules have equal probability 
of getting closer or further from the (static) solute, as expected.

Another function of interest, directly related to the former, answers the following question: of all the molecules having inward velocity, which fraction can be found at angle $\theta$ ? It will be denoted as $\wp_{+}(\theta)$ and, according to this definition, it can be found (except for a normalization factor) from the relation

$$
\left.\wp_{+}(\theta) \propto \quad \text { (fraction of molecules at angle } \theta\right) p_{+}(\theta) \text {. }
$$

The term in brackets $[n(\theta)]$ can be expressed (see Fig. 11) as

$$
n(\theta)=\frac{\text { number of molecules in a ring defined by }(\theta, \theta+d \theta) \text { and thickness } d r}{\text { number of molecules in a special shell of thickness } d r}
$$

Again, from inspection of Fig. 11, it is clear that (if $\rho$ denotes the number density)

$$
n(\theta)=\frac{\rho 2 \pi r^{2} \sin \theta d r d \theta}{\left(\int_{0}^{\pi} \sin \theta d \theta\right) \rho 2 \pi r^{2} d r}=\frac{\sin \theta}{2} d \theta,
$$

so that (in units of $\mathrm{rad}^{-1}$ )

$$
\begin{aligned}
\wp_{+}(\theta) \propto & \frac{\sin (\theta)}{2 \pi}\left\{\tan ^{-1}\left(\sqrt{\frac{m_{2}}{m_{1}}} \cos \theta\right)\right. \\
& \left.+\frac{1}{2} \sin \left[2 \tan ^{-1}\left(\sqrt{\frac{m_{2}}{m_{1}}} \cos \theta\right)\right]+\frac{\pi}{2}\right\} .
\end{aligned}
$$

Note that a proper normalization is still required as, according to its definition, it should satisfy

$$
\int_{0}^{\pi} \wp_{+}(\theta) d \theta=1
$$

If this closure relation is imposed for the simple case $m_{2} / m_{1} \rightarrow 0$, we obtain the final result

$$
\begin{aligned}
\wp_{+}(\theta)= & \frac{\sin (\theta)}{\pi}\left\{\tan ^{-1}\left(\sqrt{\frac{m_{2}}{m_{1}}} \cos \theta\right)\right. \\
& \left.+\frac{1}{2} \sin \left[2 \tan ^{-1}\left(\sqrt{\frac{m_{2}}{m_{1}}} \cos \theta\right)\right]+\frac{\pi}{2}\right\} .
\end{aligned}
$$

${ }^{1}$ A. Einstein, Ann. Phys. 17, 549 (1905).

${ }^{2}$ J. P. Hansen and I. R. McDonald, Theory of Simple Liquids (Academic, London, 1986).

${ }^{3}$ R. Walser, B. Hess, A. E. Mark, and W. F. van Gunsteren, Chem. Phys. Lett. 334, 337 (2001).

${ }^{4}$ J. T. Hynes, Annu. Rev. Phys. Chem. 28, 301 (1977); J. T. Hynes, R. Kapral, and M. Weinberg, J. Chem. Phys. 70, 871 (1979); 67, 3256 (1977); 69, 2725 (1978).

${ }^{5}$ S. Bhattacharyya and B. Bagchi, J. Chem. Phys. 106, 1757 (1997); B.
Bagchi and S. Bhattacharyya, Adv. Chem. Phys. 116, 67 (2001); S. Pal, S. Balasubramanian, and B. Bagchi, Phys. Rev. E 67, 061502 (2003).

${ }^{6}$ S. H. Lee and J. Rasaiah, J. Phys. Chem. 100, 1420 (1996).

${ }^{7}$ P. G. Wolynes, J. Chem. Phys. 68, 473 (1978); P. G. Wolynes, Annu. Rev. Phys. Chem. 31, 345 (1980), and references therein.

${ }^{8}$ R. Biswas, S. Roy, and B. Bagchi, Phys. Rev. Lett. 75, 1098 (1995); R. Biswas and B. Bagchi, J. Chem. Phys. 106, 5587 (1997); B. Bagchi and R. Biswas, Acc. Chem. Res. 31, 181 (1998).

${ }^{9}$ R. W. Impey, P. Madden, and I. R. McDonald, J. Phys. Chem. 87, 5071 (1983).

${ }^{10}$ S. Koneshan, J. C. Rasaiah, R. M. Lynden-Bell, and S. H. Lee, J. Phys. Chem. B 102, 4193 (1998).

${ }^{11}$ T. M. Chang and L. X. Dang, J. Phys. Chem. B 103, 4716 (1999).

${ }^{12}$ S. Chowdhuri and A. Chandra, J. Chem. Phys. 115, 3732 (2001).

${ }^{13}$ R. Rey and J. T. Hynes, J. Phys. Chem. 100, 5611 (1996).

${ }^{14}$ R. Rey and J. T. Hynes, J. Phys.: Condens. Matter 8, 9411 (1996).

${ }^{15}$ D. Spångberg, R. Rey, J. T. Hynes, and K. Hermansson, J. Phys. Chem. B 107, 4470 (2003).

${ }^{16}$ L. Helm and A. E. Merbach, Coord. Chem. Rev. 187, 151 (1999).

${ }^{17}$ H. J. C. Berendsen, J. P.M. Postma, W. F. van Gunsteren, and J. Hermans, in Intemolecular Forces, edited by B. Pullman (Reidel, Dordrecht, 1981).

${ }^{18}$ J. P. Ryckaert, Mol. Phys. 55, 549 (1985).

${ }^{19}$ B. M. Pettitt and P. J. Rossky, J. Chem. Phys. 84, 5836 (1986).

${ }^{20}$ L. X. Dang, J. Chem. Phys. 96, 6970 (1992).

${ }^{21}$ M. P. Allen and D. J. Tildesley, Computer Simulation of Liquids (Oxford University Press, New York, 1989).

${ }^{22}$ H. J. C. Berendsen, J. P. M. Postma, W. F. van Gunsteren, A. di Nola, and J. R. Haak, J. Chem. Phys. 81, 3683 (1984).

${ }^{23}$ A. Rahman, Phys. Rev. 136, A405 (1964).

${ }^{24}$ L. Verlet, Phys. Rev. 159, 98 (1967).

${ }^{25}$ M. Masia and R. Rey, J. Phys. Chem. B 107, 2651 (2003).

${ }^{26}$ It is, however, certainly the case that the inferences from simple kinematic arguments can be superseded by the effect of strong chemical interactions; the well-known case of the Walden inversion for $\mathrm{SN}_{2}$ nucleophilic displacement reactions provides an obvious example.

${ }^{27}$ H. L. Friedman, Chem. Scr. 25, 42 (1985).

${ }^{28}$ H. J. C. Berendsen, J. R. Grigera, and T. P. Straatsma, J. Phys. Chem. 91, 6269 (1987).

${ }^{29}$ L. Verlet, Phys. Rev. 165, 201 (1968).

${ }^{30}$ D. Levesque and L. Verlet, Phys. Rev. A 2, 2514 (1970).

${ }^{31}$ A. Chandra, J. Phys. Chem. B 107, 3899 (2003).

${ }^{32}$ B. Nigro, R. Rey, and J. T. Hynes (unpublished).

${ }^{33}$ S. Raugei and M. L. Klein, J. Chem. Phys. 116, 196 (2002).

${ }^{34}$ M. Masia, M. Probst, and R. Rey, J. Chem. Phys. 121, 7362 (2004). 